\title{
Las desigualdades en la educación secundaria argentina durante la pandemia
}

\author{
Inequalities in Argentine secondary education \\ during the pandemic
}

\section{Mariano Anderete Schwal ${ }^{*}$}

Recibido: 24 de enero de 2021 Aceptado: 28 de abril de 2021 Publicado: 27 de julio de 2021

To cite this article: Anderete Schwal, M. (2021). Las desigualdades en la educación secundaria argentina durante la pandemia. Márgenes, Revista de Educación de la Universidad de Málaga. 2 (2), 42-56

DOI: http://dx.doi.org/10.24310/mgnmar.v2i2.11638

\section{RESUMEN}

El presente trabajo analiza las consecuencias educativas de la pandemia por coronavirus en la educación secundaria argentina y los efectos que generó la segregación educativa en los docentes y el alumnado en un contexto determinado. Se trata de un estudio descriptivo, utilizando un método cualitativo a partir de entrevistas a directivos del nivel secundario de la ciudad de Bahía Blanca, Argentina. Se considera una muestra diversa, incluyendo a escuelas públicas y privadas, con distintas modalidades y ubicadas geográficamente en diferentes zonas de la ciudad, a modo de contrastar las percepciones según las distintas realidades de las instituciones. Al analizar la puesta en práctica de la educación a distancia, los testimonios dan cuenta de una brecha digital derivada de la desigualdad socio económica entre los estudiantes que asisten a distintos tipos de escuelas. Los ejes analizados giran en torno de los recursos tecnológicos de los estudiantes, la preparación de los docentes, las dificultades económicas de las familias y el abandono estudiantil en el contexto de pandemia. Los resultados dan cuenta de un aumento de la desigualdad educativa a partir del confinamiento por Covid-19.

Palabras clave: segregación educativa; seguimos educando; desigualdades; brecha digital; educación en pandemia

\section{ABSTRACT}

This work analyzes the educational consequences of the coronavirus pandemic in Argentine secondary education and the effects it had on teachers and students, in a context of educational segregation. It is a descriptive study, using a qualitative method based on interviews with high school managers in the city of Bahía Blanca, Argentina. A diverse sample is considered, including public and private schools, with different modalities and geographically located in different areas of the city, in order to contrast perceptions according to the different realities of the institutions. When analyzing the implementation of distance education, the testimonies reveal a digital gap derived from socio-economic inequality between students who attend different types of schools. The axes analyzed revolve around the technological resources of students, the preparation of teachers, the economic difficulties of families and student abandonment in the context of a pandemic. The results show an increase in educational inequality from the confinement by Covid-19.

Keywords: educational segregation; we continue educating; inequalities; digital divide; pandemic education 


\section{INTRODUCCIÓN}

La aparición de la pandemia producida por el coronavirus conocido como Covid- $19^{1}$ a principios del año 2020 generó cambios profundos en la sociedad, produciendo un aumento de la pobreza y exponiendo las desigualdades existentes a nivel mundial (CEPAL, 2020a). En tal sentido se evidenciaron las desigualdades sociales, culturales y económicas en todos los países afectados por la pandemia (Bravo García y Magis Rodríguez, 2020). La educación durante el año escolar continuó brindándose al tratarse de un derecho humano fundamental (Ruiz, 2020), pero se hizo con las escuelas cerradas por decisión de los gobiernos nacionales para evitar posibles contagios, afectando al $94 \%$ de estudiantes de todo el mundo (UNESCO, 2020). Las diferencias sociales repercutieron fuertemente en la educación a distancia propuesta, exponiéndose las desigualdades en oportunidades y condiciones (Tarabini, 2020).

Ante la aparición de la pandemia, el gobierno nacional argentino determinó la cuarentena obligatoria de todos los ciudadanos a los efectos de disminuir la curva de contagios en el país. En el ámbito educativo se presentó el desafío de poder continuar con la educación de más de diez millones de niños y adolescentes desde sus casas, sin poder ir a las escuelas. Entonces el Ministerio de Educación desarrolló un proyecto llamado "Seguimos Educando", por el cual se utilizaron principalmente recursos de internet, radio, televisión y entrega de cuadernillos con el objetivo de asegurar la continuidad educativa a distancia. Pero su aplicación no fue obligatoria sino que dependía de la voluntad de los docentes, quienes en su mayoría no lo utilizaron por diversas razones como la falta de recursos, no adaptarse a los contenidos de sus materias, considerar a la televisión un entretenimiento o cuestionar la postura política del gobierno nacional (Anderete Schwal, 2020). Debido a la segregación educativa existente en Argentina se pudieron observar distintas formas de afrontar la educación a distancia durante la pandemia, dependiendo de los recursos materiales y digitales con los que contaban las escuelas, docentes y fundamentalmente sus estudiantes.

Los profesores son los encargados y responsables de enseñarles a los alumnos cómo utilizar los recursos tecnológicos para su educación (Bonilla Guachamín, 2020). Puesto que si bien la mayoría de los adolescentes están familiarizados con la tecnología, la destinan principalmente para redes sociales y videojuegos, y no para las actividades escolares (Gewerc, Fraga y Rodés, 2017). En tal sentido los docentes debieron cumplir un nuevo rol en la enseñanza, mediatizando las herramientas didácticas mediante la virtualidad para favorecer el aprendizaje de su alumnado (García, 2020).

La educación virtual hasta el año 2020 estaba siendo desarrollada principalmente por las universidades, siendo el nivel universitario aquel más preparado para afrontar la educación a distancia (Silva, 2017). Incluso todas las universidades en Argentina ya tenían plataforma virtual propia y ofrecían contenidos que complementaban las clases presenciales (Muiños de Britos, Cambours de Danani y Lastra, 2021). Pero el resto de los niveles educativos no estaban preparados para llevar a cabo una educación no presencial, la mayoría de las escuelas no contaban con plataforma propia ni tenían experiencia en este tipo de educación. A partir de la suspensión de 
clases presenciales, toda la comunidad educativa debió adaptarse a la modalidad a distancia o virtual según el caso. Aunque no todos los actores educativos tuvieron las mismas posibilidades de adaptarse al cambio.

El presente trabajo analiza las consecuencias educativas de la pandemia Covid-19 en la educación secundaria argentina y los efectos que generó la segregación educativa en los docentes y el alumnado en un contexto. La base de la información son los testimonios de directivos del nivel secundario de la ciudad de Bahía Blanca, provincia de Buenos Aires. Las entrevistas fueron realizadas por el equipo del proyecto general de investigación llamado "Equidad educativa: Segmentación escolar en la localidad de Bahía Blanca”, de la Universidad Nacional del Sur.

\section{DESARROLLO TEÓRICO}

El presente trabajo se articula teóricamente con los conceptos de segregación educativa aplicables al sistema argentino y la brecha digital producida por el desigual acceso a las tecnologías de la información y la comunicación, dichas ideas se interrelacionan y se ponen de manifiesto conjuntamente en tiempos de pandemia.

\subsection{La segregación educativa}

La segregación educativa consiste en la distribución desigual de los estudiantes entre las escuelas de una ciudad, de manera tal que la proporción que representa cierta característica social en una institución (por ejemplo género, etnia, nivel socioeconómico, etc.) es significativamente diferente a la que se observa en la población total de referencia (Krüger, 2014). En esta investigación se considera a la segregación educativa según nivel socioeconómico del alumnado; Veleda (2014) expresa que ésta se entiende como la división del estudiantado según su nivel socioeconómico en circuitos educativos desiguales por sus experiencias de socialización, condiciones de aprendizaje y saberes que brindan.

Los primeros trabajos sobre segregación educativa en Argentina se remontan a Braslavsky (1985) y posteriormente a los trabajos de Kessler (2002), Tiramonti (2004), Gasparini y otros (2011), y recientemente Krüger (2019). Estos estudios demuestran la considerable distancia de acceso a la educación de calidad según el sector social de pertenencia. Además de determinar este acceso diferencial, destacan la existencia de los circuitos y recorridos educativos diversos que realizan los estudiantes según su identificación social y económica. Lo cual genera la conformación de grupos diferenciados que tienen pocas posibilidades de cruzarse con sus pares que transitan por distintos circuitos educativos. Más allá de que los estudiantes hayan logrado acceder al sistema o aprender lo esperado, la falta de promoción del intercambio respetuoso entre personas de diferentes estratos sociales perjudica la cohesión social y la construcción de una ciudadanía democrática (Krüger, 2019).

Se advierten dos instancias de segregación en la incorporación al sistema educativo de los estudiantes: intersectorial e intra-sectorial (Krüger, 2014). La primera se refiere a la diferenciación en el perfil socioeconómico de los estudiantes de las redes de gestión estatal y privada. Y la segunda se relaciona con la desigual composición social de las escuelas dentro de cada uno de 
estos circuitos, donde, por ejemplo, la composición social de las escuelas públicas céntricas es diversa a aquellas ubicadas en la periferia de las ciudades. Esta situación se relaciona con la segregación residencial, que puede definirse como una relación espacial vinculada a la agrupación de viviendas ocupadas por hogares que pertenecen a un mismo estrato social. Así, pueden observarse grupos de habitantes más pobres establecidos en barrios con menor cobertura de bienes y servicios, y también grupos de habitantes más privilegiados que se auto-aíslan en ciertos sectores con mayores recursos (PNUD, 2009). En el caso de Bahía Blanca coinciden los ámbitos de segregación mencionados, donde las carencias respecto a condiciones socioeconómicas se distribuyen en forma de anillos concéntricos que aumentan progresivamente desde el centro de la ciudad hacia la periferia de la concentración urbana, en coincidencia con una gradación descendente que caracteriza a varias ciudades argentinas y latinoamericanas (Prieto, 2008).

Finalmente, en las ciudades argentinas que cuentan con escuelas secundarias tradicionales dependientes de Universidades Nacionales, se advierte una marcada diferencia con el resto de las escuelas públicas provinciales. Ya que las escuelas preuniversitarias se encuentran colonizadas históricamente por una clase media con determinado capital cultural (Di Piero, 2016) y dichas instituciones educativas cuentan con mayores recursos económicos que las demás, al contar con un presupuesto universitario. Por tales causas la conformación de su alumnado se asemeja al de instituciones de gestión privada, a pesar de ser escuelas públicas.

\subsection{Tics y la Brecha Digital}

Hasta el año 2020 la educación virtual estaba reservada a experiencias aisladas dentro de las escuelas secundarias, las cuales aportaban estrategias innovadoras de enseñanza y aprendizaje aplicadas complementariamente a la educación presencial (Expósito y Marsollier, 2020). En tal sentido las Tecnologías de la Información y la Comunicación (TIC) se desarrollan hace décadas progresivamente, siendo herramientas disponibles al servicio de la educación y de los docentes (Almirón y Porro, 2014). En el contexto de la pandemia las tareas educativas del docente se modificaron y se incrementaron, en cuanto a que sus buenas prácticas son facilitadoras de los procesos educativos mediante el uso de las TIC.

Desde el punto de vista de los estudiantes las TIC en el hogar son fundamentales para posibilitar el acceso a la educación en el contexto de la cuarentena. A estos recursos digitales necesarios se les deben sumar la presencia de un adulto que oriente a los niños o adolescentes que intenten utilizarlos. Pero no todos los alumnos disponen de los mismos recursos digitales, existiendo una marcada desigualdad dependiendo de la situación socioeconómica que presente familia (Formichella y Krüger, 2020). En el caso de Argentina existe una desigual conexión a internet según el nivel socio económico (NSE) de las familias, en tanto que los estudiantes de secundaria de bajo NSE con acceso a internet es del 54,85\%, mientras los de alto nivel es del 97,88\%; la proporción es similar al considerar las viviendas con computadora de dichos estudiantes $(54,85 \%$ nivel bajo y 99,03\% nivel alto), mientras que en la tenencia de teléfonos móviles la brecha no es tan marcada, ya que el $91,80 \%$ de los estudiantes con bajo nivel cuentan con uno, así como el 98,93\% de nivel alto (Cardini, D 'Alessandre y Torre, 2020).

La Brecha Digital es un concepto creado para definir las desigualdades en el acceso y uso de las TICs. Pimienta (2007) afirma que es el reflejo de la brecha social en el mundo digital. Esta 
situación se evidencia en las diferencias en el acceso a recursos tecnológicos y a la conexión a internet, especialmente en los países latinoamericanos (CEPAL, 2020b). Esta brecha digital puede reproducirse a través del sistema educativo de diversas maneras, ya que tanto los estudiantes, como los profesores y las familias, cuentan con dificultades y limitaciones que impiden que las competencias tecnológicas y el acceso a recursos educativos se dé en condiciones igualitarias que permitan disminuir la brecha no sólo digital, sino también educativa.

Alderete y Formichella (2020) afirman que al considerar los recursos digitales de todas las familias se advierten las fuentes de desigualdad entre los hogares según sus recursos económicos, ya que no todos cuentan con la calidad de los dispositivos necesarios para realizar la diversidad de actividades escolares pretendidas; la cantidad de dispositivos en el mismo hogar puede ser insuficiente para cubrir las necesidades escolares y laborales de todos sus miembros; el acceso a Internet no es igual para todos; y las desigualdades en el acceso a las TIC de los estudiantes se vinculan con las desigualdades de sus resultados educativos.

Los organismos internacionales como UNESCO (2020) y UNICEF (2020) advierten que, además de la brecha digital entre clases sociales, existe una brecha familiar por la cual las familias con menor educación tienen más dificultades para ayudar a sus hijos e hijas en las tareas escolares domiciliarias, generando así un aumento mayor de la segregación educativa durante la cuarentena. Echeita (2020) se plantea qué sucede con aquellos menores que no tienen acceso a internet, o cuyos padres que no tienen el tiempo o la competencia necesaria para ayudarlos en sus deberes de la escuela. La educación virtual durante la pandemia parece estar pensada para familias "normales" y no tiene en cuenta las situaciones desventajosas mencionadas. Este contexto pandémico con aislamiento social, desigual acceso a la tecnología y familias con diversa disponibilidad para acompañar a sus menores escolarizados, parece producir un aumento de la segregación educativa.

\section{METODOLOGÍA}

El estudio que se presenta es de carácter descriptivo y cualitativo, el cual pretende estudiar la situación de las escuelas secundarias durante el año 2020 en el contexto de la cuarentena por Covid-19 en Argentina, específicamente en colegios de la ciudad de Bahía Blanca en un contexto de segregación educativa y diversas situaciones de aplicación de las tecnologías de la información y la comunicación. Se realizaron entrevistas a directivos de escuelas secundarias ubicadas en la ciudad de Bahía Blanca, seleccionando dentro de dicho universo dieciséis instituciones del sector público y privado.

Siguiendo a Maxwell (1996) se seleccionaron intencionalmente los sujetos a entrevistar, escogiendo a un directivo por escuela. Los niveles socioeconómicos representados fueron los siguientes: dos escuelas privadas céntricas sin subvención de nivel alto; dos escuelas preuniversitaria de nivel medio-alto; cuatro privadas subvencionadas de nivel medio; cinco públicas céntricas y barriales de nivel medio-medio bajo; y tres escuelas públicas ubicadas en la periferia de nivel bajo. En tal sentido la muestra se estructura a partir de la heterogeneidad, haciendo uso de la estratificación (Valles, 1999). Por ello las escuelas seleccionadas son lo más diversas posible; incluyendo instituciones tanto públicas como de gestión privada, y dentro de dichos grupos las 
que se ubican en el centro y en la periferia de la ciudad, así como aquellas que ofrecen distintas orientaciones, de forma tal que se pueda abarcar a escuelas cuyo alumnado represente distintas realidades sociales.

El trabajo de campo se realizó con entrevistas mediante video llamadas al personal directivo del nivel secundario de la ciudad, siendo protagonistas y a la vez testigos de las consecuencias educativas producidas durante el año de cuarentena por pandemia. A tales efectos, se elaboró una guía de entrevista semiestructurada que comprende aspectos relevantes para el presente estudio. La entrevista semiestructurada es una de las técnicas más utilizadas en la metodología cualitativa (Flick, 2004), en ellas se desarrolla una conversación entre dos o más personas, partiendo de unas preguntas previamente seleccionadas. El guion utilizado se encontraba conformado por preguntas abiertas y no era rígido, dando lugar a que no se formulen exactamente las mismas preguntas ni en idéntico orden a todos los entrevistados, pero a su vez permite comparar las respuestas, dado que hay un esquema común. Además, se proponen las preguntas de manera tal que existe cierta libertad al momento de dar las respuestas, con el fin de apreciar de forma más adecuada la perspectiva de los actores (Valles, 1999).

Las entrevistas aportan perspectivas valiosas para comprender el efecto de la pandemia en la educación secundaria. Sin embargo, esto no implica ignorar las limitaciones que tiene esta metodología de investigación (Dussel, 2009). La autenticidad de este método aparece mediada por múltiples elementos, entre ellos el uso del testimonio personal como perspectiva de narración de sí mismo (Arfuch, 1998). También podría mencionarse otra limitación, que es la tendencia de las personas entrevistadas a declarar como propias las opiniones que creen convenientes y compartidas por la mayoría. Este fenómeno muestra el peso de la cultura sobre el individuo y es conocido como "deseabilidad social” (Corbetta, 2003). Los directivos están acostumbrados a asumirse como la "voz oficial” de la escuela, siendo los portavoces ante la comunidad escolar y ante la sociedad, por lo cual el efecto de deseabilidad podría pronunciarse (Dussel, 2014). A su vez es posible esperar una variedad de respuestas por las características única que se presentan en cada institución educativa participante y cada director busca imprimirle su sello personal a su gestión.

A continuación se presentan los resultados obtenidos a partir de las declaraciones recogidas en las entrevistas, las cuales dan cuenta de la diversidad de situaciones presentadas en las distintas escuelas secundarias de Bahía Blanca durante la pandemia, en un contexto de segregación educativa vigente durante el año 2020.

\section{RESULTADOS Y DISCUSIÓN}

A partir de las entrevistas realizadas, se organizan las respuestas en torno a distintos ejes que representan las problemáticas recurrentes mencionadas y se categorizan según el nivel socioeconómico de los estudiantes alcanzados por cada institución educativa. De tal manera se reflejan diversas situaciones descriptas por el personal directivo entrevistado dependiendo del tipo de escuela que dirigen. La mirada de las autoridades escolares le aporta una perspectiva valiosa a la investigación sobre las consecuencias de la pandemia en la educación secundaria argentina y su vínculo con la desigualdad educativa. 


\subsection{Recursos tecnológicos de los estudiantes y estrategias adoptadas}

Se presentan grandes diferencias en cuanto a los recursos tecnológicos disponibles según el nivel socioeconómico de las familias que asisten a cada tipo de escuela, de tal forma que aquellas de gestión privada son las que tienen plataformas digitales propias anteriores a la pandemia y sus estudiantes cuentan en su totalidad con computadoras y acceso a internet. En la misma situación se encuentran las escuelas preuniversitarias, que atienden a sectores económicos similares. Estos colegios contaron con los recursos suficientes pudieron continuar con la enseñanza a distancia.

Nosotros tenemos lo siguiente: Una plataforma de la editorial Santillana, que lo usa todo primaria, primer año, segundo y tercero del secundario. Cuarto, quinto y sexto tiene otra plataforma que es "colegium”, pero después usan el Zoom complementariamente para comunicarse con sus alumnos. (Directivo Escuelas privada céntrica, atiende a clases altas).

Usamos la plataforma que usa la universidad, que es el moodle. Este año algunos docentes tuvieron que capacitarse para poder usarlo. De parte de los alumnos no tuvimos problemas porque todos tienen conectividad desde sus computadoras o sus celulares. (Directivo Escuela preuniversitaria, atiende a sectores medios altos)

Dentro del grupo de los sectores socio económicos medios se encuentran las escuelas secundarias privadas subvencionadas y las públicas ubicadas en el sector céntrico y en barrios de clase media. La mayor parte de sus estudiantes cuentan con acceso a internet y con computadoras o teléfonos móviles para realizar las tareas, aunque la accesibilidad no es total ya que su público es socialmente heterogéneo. Estas escuelas no cuentan con plataformas digitales propias, pero sí se ponen de acuerdo entre los directivos y el cuerpo docente para usar una plataforma de acceso público común como es Google Classroom. También suelen utilizar el correo electrónico y el Whatsapp como herramientas supletorias. Se advierte como diferencia que las escuelas privadas proponen el dictado de clases sincrónicas, donde los docentes se conectan al mismo momento que sus estudiantes para explicar la lección, mientras que las públicas optan por las clases asincrónicas.

Con los alumnos estamos trabajando a través de la plataforma classroom de google, la cuestión de las aulas virtuales, de llevar el trabajo académico. Y después hemos ido generando distintos espacios de encuentro y compartir que han tenido que ver con las clases sincrónicas que los docentes van proponiendo. La gran mayoría va optando por algún espacio de encuentro porque sabemos que esto es necesario. (Directivo Escuela privada céntrica, atiende a clases medias)

Nosotros teníamos un Facebook escolar de noticias funcionando, pero para dar clases se habilitaron classroom, hubo docentes que les funcionó, hubo docentes que fue un fracaso total. Algunos crearon grupos de Whatsapp, gente que siguió con el classroom, que habilitaron una casilla de mail, y también tuvimos gente con cuadernillo que no tenían ningún tipo de conexión. (Directivo Escuela pública céntrica, atiende a clases medias) 
En el caso de las escuelas públicas ubicadas en la periferia pobre de la ciudad (Chavez, 2017), se evidencia la brecha digital. Donde la mayor parte de los estudiantes tiene recursos tecnológicos limitados, lo cual dificulta las posibilidades de estar conectados a través de internet desde sus casas.

La verdad es que todos se han adaptado muy bien, hemos buscado un montón de estrategias para que todos los chicos tengan continuidad pedagógica. Hay profes que trabajan con classroom, pero bueno la realidad es que no todos los chicos tienen acceso al classroom. Entonces se implementó el mail, después los grupos de whatsapp, que es lo que más funciona. Lo que más manejan los chicos y los profes son los grupos de whatsapp. Después vinieron los cuadernillos para aquellos que no tenían conectividad, pero los cuadernillos proponen actividades que no son las mismas que están desarrollando los profesores. Entonces descartamos los cuadernillos y estamos trabajando con una fotocopiadora que está a la vuelta de la escuela, entonces los chicos que no tienen conectividad van y buscan las tareas en la fotocopiadora. Y aquellos que no tienen dinero que para pagarlas, las paga la cooperadora. (Directivo escuela pública de la periferia, atiende a clases bajas)

No todos los estudiantes de los barrios más pobres cuentan con un ordenador, por lo que las tareas son comunicadas por los profesores y enviadas por el alumnado mediante la aplicación Whatsapp de sus teléfonos móviles, las cuales son realizadas a mano y presentadas a través de fotografías, otra alternativa es dejarles copias en papel de las clases y actividades a realizar, las cuales retiran los días de entrega de bolsones de comida. Hay consenso entre los entrevistados que los cuadernillos oficiales del programa "Seguimos Educando" no son utilizados por los docentes.

De los 728 alumnos solo 10 cuadernillos entregamos, porque es diferente, el cuadernillo... Nosotros no estamos de acuerdo con el cuadernillo porque no son los contenidos para ellos. El cuadernillo para cuarto, quinto y sexto año es el mismo. Entonces no sé quién los ha ideado, pero no es la idea que tenemos nosotros de libertad. Por eso te digo, no son los temas que tiene la escuela pensada. La escuela está pensada para que ellos puedan pensar y discernir por sí solos. (Directivo escuela pública periferia, atiende a clases bajas)

Tal como sucedió en el nivel primario, el programa oficial de educación a distancia no fue aplicado por los docentes (Anderete Schwal, 2020). Los motivos son diversos, en este caso la directora explica los defectos en los contenidos pedagógicos abarcados, pero también da a entender una contradicción ideológica con la propuesta oficial.

\subsection{Preparación de los docentes}

El personal docente que trabaja en las secundarias argentinas es socialmente homogéneo, ya que la mayoría pertenece a clases medias (Kessler, 2002), no obstante al preparar sus clases deben adaptarse a las diversas características de cada escuela y su alumnado.

La educación a distancia durante la pandemia significó un gran desafío para los profesores, ya que estaban acostumbrados a dictar sus clases en forma presencial y debieron pasarse al plano virtual. Los directivos tuvieron que unificar la forma de dar las clases virtuales en cada escuela y también capacitar al personal docente que no sabía cómo hacerlo. 
El primer mes fue muy difícil porque hubo que modificar la reforma de trabajo abruptamente y hubo una brecha generacional, quienes tienen 40 años o menos están acostumbrados a trabajar online y no les costó adaptarse, y quienes son más grandes de 40 a 60 años fue una barrera. No tanto el acceso a internet, porque no tenemos docentes que hayan manifestado que no tienen computadora o internet pero sí poder trabajar de forma diferente. Por eso muchos docentes lo primero que usaron fue whatsapp o mail, y hubo resistencia a usar aula virtual. Pero hubo reuniones para que los docentes puedan adaptarse a esta nueva forma de trabajo. No tiene que ver tanto con la falta de recursos, sino con qué hacer con los recursos que tenemos. Con el paso de los meses se fueron armando clases en classroom. (Directivo Escuela pública céntrica, atiende a clases medias).

Los docentes mucho más jóvenes estaban a tono con lo tecnológico. Otros se sumaron y se adaptaron. Y hay un segmento de docentes que sigue siendo reticente a utilizar todos los medios. En el caso de mi escuela siempre la capacitación ocupó un espacio muy importante, nosotros con el docente cuando se incorpora a la escuela su mail pasa a ser parte de la plataforma donde nosotros tenemos contactos, donde cada cosa nueva que recibimos se la bajamos por mail. Permanentemente les enviamos lo que se recibe de capacitación. Se ha estado insistiendo en las capacitaciones que tienen que ver con lo tecnológico. (Directivo de escuela pública Técnica, atiende a clases medias-bajas).

Se destaca una diferencia generacional entre los docentes más jóvenes - que dominan el uso de internet - y aquellos próximos a jubilarse que presentaron mayores resistencias al no estar acostumbrados a usar la computadora como herramienta de trabajo. Asimismo la adaptación del personal docente fue gradual a lo largo del año escolar, comenzando por los recursos más conocidos como el Whatsapp y el correo electrónico, hasta que finalmente adaptaron una plataforma virtual en común, que en el caso de las escuelas públicas céntricas fue mayormente el Google Classroom y en las privadas la plataforma propia de la escuela.

Eso nos fue acomodando con el tema de acercarnos a una sola plataforma. Yo me puse muy exigente con que sea una sola plataforma. Además con Moodle no se gastan datos, no es como classrooom. Eso nos costó un montón de enojos de algunos profesores que no estaban acostumbrados. Yo los entendí, pero bueno igual tuve mucho apoyo de los profes de informática que ayudaron con Moodle. (Directivo Escuela preuniversitaria, atiende a sectores medios altos)

En el caso de las escuelas secundarias preuniversitarias, que son aquellas dependientes de la Universidad Nacional del Sur, se utilizó la plataforma virtual Moodle que utiliza la universidad. Todos los directivos entrevistados enfatizaron la necesidad de capacitar al personal docente para que aprendan a utilizar la nueva tecnología.

Le fuimos buscando la vuelta. Hay profes que tienen que enseñar en inglés también, tenemos italiano. Lo importante de esto fue que tratamos de buscar por todos los costados lo positivo. Los docentes estuvieron bien, todo el tiempo con los docentes, el psicólogo y el equipo directivo todo el tiempo detrás de los docentes, el colegio todo el tiempo, si tenían dispositivos, si tenían la cámara, si tenían una computadora, si se les rompían íbamos al colegio y le buscábamos una nueva. Cuando fue el día del maestro, el equipo directivo salió a repartir un regalito a todos ellos, a todos los docentes. Fuimos a las casas, no lo podían creer. (Directivo Escuela privada céntrica, atiende a clases altas) 
Las escuelas pertenecientes a sectores de clase alta se caracterizan por tener una fuerte presencia y seguimiento de su plantel docente, es así que al momento de dictar las clases a distancia les brindaron todos los recursos tecnológicos posibles para poder cumplir con su tarea. Los directivos no mencionaron problemas de capacitación docente debido a que dichas escuelas ya trabajaban con actividades escolares a distancia y su personal ya estaba preparado para aplicarlas.

\subsection{Dificultades económicas de las familias}

La cuarentena dispuesta por el gobierno argentino implicó una paralización casi total del país, con consecuencias económicas negativas para la mayoría de sus habitantes. Esta crisis se vio reflejada en las escuelas, aunque la clase media alta pudo absorber el impacto y las escuelas privadas donde estudian intentaron contener a aquellos que no podían pagar su cuota, a los efectos de mantener su matrícula.

Todo se basa en los aportes de las familias, de la cuota. Este año fue difícil, porque hubo mucha baja en maternal y mucho papá con problemas para pagar la cuota, lo cual el colegio estuvo muy al lado de las familias. E hizo, digamos, reducciones. Atendió todos los casos y ninguno se quedó afuera. Muchas familias que tienen negocio, médicos que no tenían consultorio, de todo. (Directivo Escuela privada céntrica, atiende a clases altas)

Muchos se pusieron en contacto por situaciones económicas, por situaciones particulares, se los acompañó en los casos de que un chico pueda quedar afuera de la escuela por razones económicas. La escuela becó a muchos estudiantes que no estaban contemplados. Las familias tienen mucho contacto con los preceptores, con los equipos de gestión. (Directivo Escuela privada de barrio, atiende a clases medias)

Mientras que los sectores de clase media y alta pudieron aguantar la presión económica que les significó la cuarentena y la paralización total de actividades, dicha paralización afectó a quienes tenían una situación laboral de mayor vulnerabilidad (Ernst et al., 2020).

Nuestra escuela no está incorporada a ninguno de los sistemas de alimentación propuestos por la provincia, ni la merienda ni los almuerzos. Y así que bueno, en una primera instancia los profes y las profes hicimos una donación, se pidió donaciones en la escuela, la verdad que todos respondieron increíblemente bien. Después hicimos aportes que también hicieron los docentes de verduras, que lo trabajamos a través de una ONG. Y después el Consejo Escolar nos entregó bolsones que contienen una porquería, por decirlo así, lo que viene. Pero para muchas de las familias era una ayuda extraordinaria. Y desde mayo, fines de mayo, estamos con la entrega de bolsones y dos por tres en el medio hacemos una compra de verduras solidaria por parte de los y las docentes. (Directivo escuela pública técnica, atiende a clases medias bajas)

Nosotros empezamos con un servicio alimentario escolar que se da en la presencialidad que consiste en una vianda, un sanguchito, una manzana que se le da en la escuela. Nosotros trabajamos con el Equipo de Orientación Escolar y se da a los chicos en situación más vulnerable económicamente. Al inicio del aislamiento se mantuvo a esos mismos chicos para hacerle entrega de un bolsón de alimentos 
que provee el consejo escolar, nosotros lo armamos en la escuela porque llega la mercadería a granel y se los damos a las familias. Empezamos con 20 o 22 familias que estaban recibiendo este bolsón de alimentos, a medida que esto fue avanzando notamos que empezaron a aparecer necesidades en otras familias que no estaban alcanzadas y hoy estamos en 168 bolsones de alimentos. O sea que cada 15 días nosotros hacemos un relevamiento nuevo y volvemos a informar y en base a ese relevamiento nos llega la mercadería. Cada 15 días nos fueron sumando familias. (Directivo escuela pública técnica, atiende a clases medias)

Nosotros no tenemos comedor, ni siquiera tenemos cocina. Ahora sí estamos entregando los bolsones de alimentos, pero no tenemos comedor. Si bien hay chicos con necesidades, se nota más ahora, antes no era tanto. (Directivo escuela pública de la periferia, atiende a clases bajas)

En el caso de las escuelas públicas de los barrios más pobres, la entrega de bolsones de comida cada quince días significó una fuente alimenticia no solo para el estudiante que solía utilizar el comedor escolar, sino para toda su familia. Entonces esa situación se transformó en un vínculo directo de la escuela con las familias, donde a medida que se prorrogaba la cuarentena se iban sumando más pedidos de bolsones. Este momento también se aprovechó para entregarles los cuadernillos impresos del proyecto "Seguimos Educando".

La desigualdad está basada en recursos económicos, no es lo mismo trabajar con un grupo de alumnos a los que podés pedirle un montón de material que estar con un chico que en su casa no tiene quién los ayude, porque los padres no tienen educación, no tienen ni la primaria. Y cuando te ponés a analizarlo te da bronca, porque es injusto. Porque los profes son los mismos, los que trabajan acá que los que trabajan en la Media 3 (escuela pública céntrica de Bahía Blanca). No sé por qué esa diferencia en calidad educativa. Sí en una cuestión económica en principio, después no sé. Por allí los chicos de otras escuelas salen mejor preparados que los de escuelas periféricas. Por ahí radica en la expectativa que ellos mismos tienen de su futuro, de su proyecto educativo. Hay chicos que te dicen esto no lo puedo hacer, no es para mí. Ellos están con la autoestima muy baja. (Directivo escuela pública de la periferia, atiende a clases bajas)

Los directivos de las escuelas ubicadas en sectores más pobres reconocen a las dificultades económicas de las familias como determinantes al momento de medir la respuesta educativa de los estudiantes. Ya que aquellos que provienen de sectores vulnerables se encuentran con numerosas problemáticas sociales para lograr superar el nivel secundario (Langer y Esses, 2019), tornándose injusta su comparación con aquellos provenientes de sectores de clase media o alta, quienes tienen un porcentaje de egreso considerablemente mayor.

\subsection{Abandono escolar}

Los colegios privados se caracterizan por tener muy poco abandono estudiantil. En el marco de la pandemia los directivos destacaron que la comunicación con la familia es inmediata cuando advierten que el alumno no se conecta. En esta situación también se encuentran los colegios preuniversitarios. 
El ausentismo y repitencia son casos aislados. Siempre se los acompaña. Cuando los profesores ven que un alumno no se conecta o no está presentando, se ponen en contacto con las familias. Ahí se comienza el camino de seguimiento con cada familia. (Directivo Escuela privada céntrica, atiende a clases medias-altas).

La tasa de abandono es mínima, nada. Hoy en día el abandono escolar en escuelas de gestión privada es casi inexistente. Se trabaja mucho en todo lo que tiene que ver la prevención de este aspecto y lamentablemente estos chicos que están ahí en el borde de abandonar, el paso previo es el pase de gestión privada a estatal y ahí los perdemos. (Directivo Escuela privada de barrio, atiende a clases medias).

Nosotros nos planteamos mantener el vínculo, crear espacios de contacto y comunicación. Como tenemos asignada una auxiliar docente por curso, eso te da la posibilidad de hacer un acompañamiento más minucioso, más específico. Al no tener contacto con un estudiante, hubo que recurrir a la visita a la casa. No en el sentido de que no abandonen, sino ver qué está pasando con un alumno. (Directivo Escuela preuniversitaria, atiende a clases medias altas).

Por otra parte, los directivos de escuelas públicas mencionan que la continuidad educativa fue afectada, discontinuándose para gran parte de su alumnado ante la imposibilidad de continuar con las clases a distancia. Destacaron que también los afecta el desánimo por una situación desfavorable educativamente que se prolongó durante todo el año escolar.

La continuidad fue afectada. Si tengo que hacer una evaluación de cuánto están aprendiendo los chicos, digo un 50\% de lo que estarían haciéndolo en contexto normal. La continuidad pedagógica afectó muchísimo. Ahora no los podemos evaluar, sí se mandan las tareas, los trabajos, los videos, pero vos no sabés cuánto aprendió. Si lo ayudaron, si lo que está haciendo lo entiende. Es muy difícil evaluar el aprendizaje en este contexto, hasta que no volvamos no lo vamos a saber. Tenés dos factores, los chicos están cansados y no quieren hacer nada, incluso los que tienen conectividad. Están como negados, después de las vacaciones de invierno se bajonearon un montón. (Directivo escuela pública periférica, atiende a clases bajas).

Este año se ve muchísimo más abandono, tenemos muchos alumnos trabajando. Por necesidad, por cuestión de que tienen tiempo. Son chicos de quinto y sexto. Hay una necesidad de trabajar para ayudar a sus familias. La repitencia reiterada también genera cierto desánimo y abandonan. (Directivo escuela pública céntrica, atiende a clases medias)

Asimismo manifiestan que los colegios públicos padecen del abandono estudiantil en mayor proporción que el resto, ocasionado por las necesidades económicas derivadas de la pandemia, por la cual aquellos que cursan en los últimos años deben salir a trabajar para ayudar a sus familias.

\section{CONCLUSIONES}

El sistema educativo argentino está signado por una segregación educativa muy marcada, la cual divide la calidad educativa impartida de acuerdo a las escuelas y a los recursos económicos de los estudiantes que allí concurren. Las desigualdades entre la educación pública y privada es replicada dentro de dichos sectores, generándose una segregación intra-sectorial donde una 
escuela pública del centro cuenta con mejores condiciones que la ubicada en la periferia. Es así que aquellos con mayores recursos cuentan con mayores posibilidades educativas. Formichella y Krüger (2013) indican que en Argentina las escuelas de gestión privada obtienen mejores resultados que las públicas porque albergan estudiantes con condiciones más favorables para hacer frente a su carrera escolar, situación que se magnifica en el contexto analizado.

La desigualdad escolar se replica y se intensifica durante tiempos de pandemia, donde la educación a distancia digital es factible para algunos sectores que tienen los recursos tecnológicos necesarios y se vuelve más difícil de lograr para otros que carecen de dichos recursos, profundizando las desigualdades iniciales.

Los directivos de las escuelas secundarias consensuaron con sus docentes las herramientas educativas a utilizar según las características socio-económicas de su alumnado. Se trató de una adaptación para lograr los mejores resultados posibles, al analizarlos se evidencian las desigualdades entre tipos de escuelas, puesto que algunas utilizaron plataformas de internet propias donde se conectaban en línea la totalidad de sus estudiantes con sus profesores, mientras que en otras los docentes debían dejar fotocopias que las familias retiraban junto con los bolsones de comida.

En cuanto proyecto oficial de educación a distancia denominado "Seguimos Educando", el mismo no cumplió con sus objetivos como herramienta educativa durante el confinamiento compulsivo. En la práctica los docentes advirtieron diversas dificultades al momento de aplicarlo, por lo cual utilizaron estrategias propias de educación a distancia.

Se propone a la tecnología como una herramienta superadora de la falta de presencialidad escolar derivada de la pandemia. Pero en un país con una desigualdad educativa y una brecha digital tan grandes, la educación a distancia no puede más que replicar dicha desigualdad si no se toman medidas complementarias tendientes a reducirla.

\section{REFERENCIAS}

Anderete Schwal, M. (2020). Las desigualdades educativas durante la pandemia en la educación primaria de Argentina. Revista Andina De Educación, 4(1), 5-10. https://doi.org/10.32719/26312816.2021.4.1.1

Arfuch, L. (1998). La entrevista, una invención dialógica. Paidós.

Almirón, M. E., y Porro, S. (2014). Los docentes en la Sociedad de la Información: reconfiguración de roles y nuevas problemáticas. IE Comunicaciones: Revista Iberoamericana de Informática Educativa, (19), 17-31. (http://bit.ly/3c5LWBR)

Braslavsky, C. (1985). La discriminación educativa en Argentina. FLACSO.

Bravo-García, E., y Magis-Rodríguez, C. (2020). La respuesta mundial a la epidemia del COVID-19: los primeros tres meses. Boletín sobre COVID-19 Salud Pública y Epidemiología, 1(1), 3-8. (https://bit. ly/3qQVZyy)

Bonilla-Guachamín, J. A. (2020). Las dos caras de la educación en el COVID-19. CienciAmérica, 9(2), 8998. http://dx.doi.org/10.33210/ca.v9i2.294 


\section{N V E S T I G A C I O N E S}

Cardini, A., D 'Alessandre, V. y Torre, E. (2020). Educar en tiempos de pandemia. Respuestas provinciales al COVID-19 en Argentina. CIPPEC.

CEPAL (2020a). COVID19 tendrá graves efectos sobre la economía mundial e impactará a los países de América Latina y el Caribe. Comunicado de Prensa. CEPAL. (http://bit.ly/2Mf1ati)

CEPAL. (2020b). Las oportunidades de la digitalización en América Latina frente al Covid-19. CEPAL. (https:/l bit.ly/3qFBfKi)

Chavez, M. (2017). Jóvenes entre el centro y la periferia de la ciudad, del estado y de la academia. CiudaDanías. Vol 1, № 6, 7.1-18. (http://bit.ly/3byFLWz)

Corbetta, P. (2003). Metodología y técnica de la Investigación Social. McGraw Hill.

Di Piero, E. (2016). Igualitarismo, mérito y nociones de justicia en torno al método de admisión a una escuela secundaria universitaria. Archivos de Ciencias de la Educación, 10(10). (https://bit.ly/3iLVi6X)

Dussel, I. (2009). La cultura escolar en contextos fragmentados: notas para pensar sobre qué puede una escuela. Mimeo.

Dussel, I. (2014). ¿Qué lugar tiene la escuela media en la producción de la desigualdad? Elementos para el debate”. Archivos Analíticos de Políticas Educativas, 22(43). http://dx.doi.org/10.14507/epaa. $\underline{\mathrm{v} 22 \mathrm{n} 43.2014}$

Echeita, G. (2020). La Pandemia del Covid-19. ¿Una Oportunidad para Pensar en Cómo Hacer más Inclusivos Nuestros Sistemas Educativos? Revista Internacional de Educación para la Justicia Social. 9(1). 7-16. https://doi.org/10.15366/riejs2020.9.3

Ernst, C., Mourelo, E., Pizzicannella, M., Rojo, S. y Romero, C. (2020). Argentina. Los retos en las respuestas a la pandemia y sus impactos socioeconómicos. Nota técnica país. OIT Américas. (https:// bit.ly/36pXDzN)

Expósito, E., \& Marsollier, R. (2020).Virtualidad yeducación en tiempos de COVID-19. Un estudio empírico en Argentina. Educación y Humanismo, 22(39), 1-22. https://doi.org/10.17081/eduhum.22.39.4214

Formichella, M. y Krüger, N. (2013). El fracaso escolar en el nivel medio argentino: ¿Es menos frecuente en las escuelas de gestión privada debido a su administración? Regional and Sectoral Economic Studies, 13(3), 127-144. (https://bit.ly/3pivDoQ)

Formichella, M. y Krüger, N. (2020). Pandemia y brechas educativas: reflexiones desde la Economía de la Educación. La investigación en ciencias sociales en tiempos de la pandemia por Covid-19. IIESS, 168-186. (https://bit.ly/2Y9HycO)

García, M. D. (2020). La docencia desde el hogar. Una alternativa necesaria en tiempos del COVID 19. Polo del Conocimiento: Revista científico-Académica Multidisciplinaria, 5(4), 304-324. https://doi. org $/ 10.23857 /$ pc.v5i4.1386

Gasparini, L., Jaume, D., Serio, M. y Vázquez E. (2011). La segregación entre escuelas públicas y privadas en Argentina. Reconstruyendo la evidencia. Revista Desarrollo Económico, 51(202), 35-57. (https://bit. $1 \mathrm{y} / 2 \mathrm{Y} 7 \mathrm{vYPt})$

Gewerc, A., Fraga, F. y Rodés, V. (2017). Niños y adolescentes frente a la Competencia Digital. Entre el teléfono móvil, youtubers y videojuegos. Revista Interuniversitaria de Formación del Profesorado, 89 (31.2), 171-186. (https://bit.ly/36aEe5z)

Kessler, G. (2002). La experiencia escolar fragmentada. Estudiantes y docentes en la escuela media en Buenos Aires. IIPE-UNESCO. 


\section{N V E S T I G A C I O N E S}

Krüger, N. (2014). Más allá del acceso: segregación social e inequidad en el sistema educativo argentino. Cuadernos de Economía, 33(63), 513-542. https://doi.org/10.15446/cuad.econ.v33n63.45344

Krüger, N. (2019). La segregación por nivel socioeconómico como dimensión de la exclusión educativa: 15 años de evolución en América Latina. Archivos Analíticos de Políticas Educativas, 27(8), 35-67. https://doi.org/10.14507/epaa.27.3577

Langer, E. y Esses, J. (2019). La salida es por arriba. Una historia de juventud, pobreza y educación. Grupo Editor Universitario.

Maxwell, J. (1996). Qualitative research design. An Interactive Approach. Sage Publications.

Muiños de Britos, S., Cambours de Donini, S. y Lastra, K. (2021). Desafíos en el escenario de la pandemia en universidades del conurbano bonaerense. From face-to-face to online university teaching. Revista de Educación. 286-264. (https://bit.ly/3c7RSKz).

Pimienta, D. (2007). Brecha digital, brecha social, brecha paradigmática. Funredes.

PNUD. (2009). Aportes para el desarrollo humano en Argentina 2009. PNUD, Buenos Aires.

Prieto, M. (2008). Fragmentación socio-territorial y calidad de vida urbana en Bahía Blanca. Geograficando: Revista de Estudios Geográficos, 4(4), 1-22. (https://bit.ly/3ovjk8p)

Ruiz, G. (2020). Marcas de la pandemia: El derecho a la educación afectado. Revista internacional de educación para la Justicia Social. 9(3), 45-59. https://doi.org/10.15366/riejs2020.9.3.003

Silva, J. (2017). Un modelo pedagógico virtual centrado en las E-actividades. Revista de Educación a Distancia, 17(53), 1-20. (http://bit.ly/39YHBOH)

Tarabini, A. (2020) ¿Para qué sirve la escuela? Reflexiones sociológicas en tiempos de pandemia global. Revista de Sociología de la Educación-RASE, 13(2), 145-155. https://doi.org/10.7203/RASE.13.2.17135

Tiramonti, G. (2004) La trama de la desigualdad educativa. Mutaciones recientes en la escuela media. Manantial.

UNESCO (2020). Consecuencias negativas del cierre de las escuelas. https://es.unesco.org/covid19/ educationresponse/consecuencias

UNICEF (2020). La educación frente al Covid-19. Propuestas para impulsar el derecho a la educación durante la emergencia. https://www.unicef.es/educa/biblioteca/la-educacion-frente-al-covid-19

Valles, M. (1999). Técnicas cualitativas de investigación social. Reflexión metodológica y práctica profesional. Síntesis.

Veleda, C. (2014). Regulación estatal y segregación educativa en la Provincia de Buenos Aires. CIPPEC. 